\title{
Attribute Reduction in Interval and Set-Valued Decision Information Systems
}

\author{
Hong Wang ${ }^{1}$, Hong-Bo Yue ${ }^{1}$, Xi-E Chen ${ }^{2}$ \\ ${ }^{1}$ College of Mathematics and Computer Science, Shan'xi Normal University, Linfen, China \\ ${ }^{2}$ Shanxi Coal Mining Adminstrators College, Taiyuan, China \\ Email:whdw218@163.com
}

Received September 6, 2013; revised October 6, 2013; accepted October 13, 2013

Copyright (c) 2013 Hong Wang et al. This is an open access article distributed under the Creative Commons Attribution License, which permits unrestricted use, distribution, and reproduction in any medium, provided the original work is properly cited.

\begin{abstract}
In many practical situation, some of the attribute values for an object may be interval and set-valued. This paper introduces the interval and set-valued information systems and decision systems. According to the semantic relation of attribute values, interval and set-valued information systems can be classified into two categories: disjunctive (Type 1) and conjunctive (Type 2) systems. In this paper, we mainly focus on semantic interpretation of Type 1 . Then, we define a new fuzzy preference relation and construct a fuzzy rough set model for interval and set-valued information systems. Moreover, based on the new fuzzy preference relation, the concepts of the significance measure of condition attributes and the relative significance measure of condition attributes are given in interval and set-valued decision information systems by the introduction of fuzzy positive region and the dependency degree. And on this basis, a heuristic algorithm for calculating fuzzy positive region reduction in interval and set-valued decision information systems is given. Finally, we give an illustrative example to substantiate the theoretical arguments. The results will help us to gain much more insights into the meaning of fuzzy rough set theory. Furthermore, it has provided a new perspective to study the attribute reduction problem in decision systems.
\end{abstract}

Keywords: Interval and Set-Valued Information Systems; Fuzzy Preference Relation; Interval and Set-Valued Decision Information Systems; Fuzzy Positive Region; Dependency Degree; Significance Measure

\section{Introduction}

Rough set theory, introduced by Pawlak in 1982, is a useful mathematic approach for dealing with uncertain, imprecise and incomplete information [1]. It has attracted much attention from researchers.

In Pawlak's original rough set theory, partition or equivalence (indiscernibility) relation is an important and primitive concept. But partition or equivalence relation is still restrictive for many applications. It is unsuitable for handing incomplete information systems or incomplete decision systems. To address this issue, several interesting and meaningful extensions to equivalence relation have been proposed in the past, such as tolerance relations [2-5], dominance relations [6] and others [7-19]. Particularly, in 1990, Dubois and Prade combined fuzzy sets with rough sets in a fruitful way by defining rough fuzzy sets and fuzzy rough sets.

Fuzzy rough sets were first proposed by Dubois and Prade to extend crisp rough set models [20,21]. Fuzzy rough sets encapsulate the related but distinct concepts of vagueness and indiscernibility, both of which occur as a result of knowledge uncertainty. Fuzzy rough set models have been a popular topic in recent years. In this paper, we introduce the interval and set-valued information systems and decision systems. Interval and set-valued information systems are important type of data tables, and generalized models of set-valued information systems and interval-valued information systems. Several authors have studied about interval and set-valued information systems. Lin et al. [6] introduced interval and set-valued information systems and presented a dominance-based rough set model for the interval and set-valued information systems. However, interval and set-valued information systems have not been investigated under the framework of fuzzy rough set model. The main objective of this paper is to introduce a fuzzy rough set model for interval and set-valued information systems by defining a fuzzy preference relation for interval and set-valued information systems. 
In rough set theory, an important concept is attribute reduction [2,13,14,16-18,22,23], which can be considered as a kind of specific feature selection. In other words, based on rough set theory, one can select useful features from a given data set. Recently, more attention has been focused on the area of attribute reduction and many scholars have studied attribute reduction based on fuzzy rough sets [2,16-18,22,23]. Dai et al. [2] proposed a fuzzy rough set model for set-valued data and investigated the attribute reduction in set-valued information systems based on discernibility matrices and functions. Yao et al. [16] proposed an attribute reduction approach based on generalized fuzzy evidence theory in fuzzy decision system. Shen et al. [17] studied an attribute reduction method based on fuzzy rough sets. Hu et al. [18] also proposed an attribute reduction approach by using information entropy as a tool to measure the significance of attributes. Rajen B. Bhatt and M. Gopal [22] put forward the concept of fuzzy rough sets on compact computational domain based on the properties of fuzzy tnorm and t-conorm operators and build improved feature selection algorithm. Zhao et al. [23] revisited attribute reductions based on fuzzy rough sets, and then presented and proved some theorems which describe the impacts of fuzzy approximation operators on attribute reduction. However, attribute reduction based on fuzzy rough set in interval and set-valued decision information systems has not been reported. In this paper, a fuzzy preference relation is defined and the upper and lower approximations of decision classes based on the fuzzy preference relation are given. Moreover, the definition of the significance measure of condition attributes and the relative significance measure of condition attributes are given in interval and set-valued decision information systems by the introduction of fuzzy positive region and the dependency degree. And on this basis, a heuristic algorithm for calculating fuzzy positive region reduction in interval and set-valued decision information systems is given.

The remainder of this paper is organized as follows. In Section 2, we give a brief introduction to interval and set-valued information systems and fuzzy preference relation. In Section 3, we propose a fuzzy rough set model for interval and set-valued information systems by defining a new fuzzy preference relation. In Section 4, fuzzy positive region reduction in interval and set-valued decision information systems is introduced into interval and set-valued decision information systems. To substantiate the theoretical arguments, an illustrative example is given in Section 5. In Section 6, we conclude this paper.

\section{Preliminaries}

\subsection{Interval and Set-Valued Information Systems}

As a result of limitation of subjective and objective con- ditions and the interference of random factors, people often get an approximation of the data in data acquisition of the data in data acquisition. In an information system, it may occur that some of the attribute values for an object are similar, we often make it difficult to determine the similar values. Therefore, sometimes there are some object's attribute values in information systems can not be determined, but we can know the range, which leads to the interval and set-valued information systems.

Definition 2.1.1. [6] Let $P$ and $Q$ are ordinary sets, if the range of variable $R$ takes set $P$ as the lower limit, set $Q$ as the upper limit, then the variable $R$ is called interval and set-valued variable.

Definition 2.1.2. [6] An information system is a quadruple $S=(U, A, V, f)$, where the universe $U$ is a nonempty finite set of objects, $A$ is a non-empty finite set of attributes, $V$ is the union of attribute domains $\left(V=\bigcup_{a \in A} V_{a}\right), V_{a}$ is the set of all possible values for attribute $a$ and $V_{a}$ is an interval and set-valued variable, $f: U \times A \rightarrow V$ is a function that assigns particular values from attribute domains to objects, then the information system is called an interval and set-valued information system.

The semantics of interval and set-valued information systems have been studied by different approaches, which, actually, fall into two types [6]:

Type 1: $\forall x \in U, a \in A$, the value of attribute $a$ for object $x$ is denoted by $V_{f_{a}^{-}(x)}^{f^{+}(x)}$, where $f_{a}^{-}(x)$, $f_{a}^{+}(x)$ are the finite sets, and satisfy condition:

$$
\begin{gathered}
\min \left(f_{a}^{+}(x)\right) \geq \max \left(f_{a}^{-}(x)\right) \\
\min \left(f_{a}^{-}(x)\right) \leq V_{f_{a}^{-}(x)}^{f^{+}(x)} \leq \max \left(f_{a}^{+}(x)\right),
\end{gathered}
$$

i.e. the minimum of set $f_{a}^{+}(x)$ at least equal to the minimum of set $f_{a}^{-}(x)$, at most equal to the maximum of set $f_{a}^{+}(x)$. The value of attribute $a$ for object $x$ is interpreted disjunctively. For example, if $a$ denotes the environmental risk assessment index of enterprise investment, then $V_{f_{a}^{-}(x)}^{f_{a}^{+}(x)}=V_{\{1\}}^{\{2,3\}}$ can be interpreted as: the lowest grade of the environmental risk assessment index is 1 , the highest grade may be 2 or 3 .

Type 2: $\forall x \in U, a \in A$, the value of attribute $a$ for object $x$ is denoted by $V_{f_{a}^{-}(x)}^{f^{+}(x)}$, where

$f_{a}^{-}(x), f_{a}^{+}(x)$ are the finite sets, and satisfy conditions: $f_{a}^{-}(x) \subseteq V_{f_{a}^{-}(x)}^{f^{+}(x)} \subseteq f_{a}^{+}(x)$ i.e. the minimum of set $V_{f_{a}^{-}(x)}^{f^{+}(x)}$ at least contains $f_{a}^{-}(x)$, at most equal to $f_{a}^{+}(x)$. The value of attribute $a$ for object $x$ is interpreted conjuctively. For example, if $a$ denotes the oral expression 
ability, then $V_{f_{a}^{-}(x)}^{f_{a}^{+}(x)}=V_{\{\text {English }\}}^{\{\text {English,French,German }\}}$ can be interpreted as: $x$ can speak English, but may also speak French and German. Therefore, the value of $V_{\{\text {English }\}}^{\text {EEnglisench,German }\}}$ may be $\{$ English $\}$, EEnglish, French $\}$, \{English, German $\}$ or English, French, German\}.

In this paper, we mainly focus on semantic interpretation of Type 1.

\subsection{Fuzzy Preference Relation}

Definition 2.2.1. [7-12] A fuzzy preference relation $\tilde{R}$ on a set of $U$ is a fuzzy set on the product set $U \times U$, which is characterized by a membership function: $\tilde{R}: U \times U \rightarrow[0,1]$. If the cardinality of $U$ is finite, the preference relation can also be conveniently represented by a $n \times n$ matrix $M(\tilde{R})=\left(r_{i j}\right)_{n \times n}$, where $r_{i j}=\tilde{R}\left(x_{i}, x_{j}\right), \quad r_{i j} \in[0,1] . \quad r_{i j}$ is interpreted as the preference degree of the $x_{i}$ over $x_{j}$. $r_{i j}=\frac{1}{2}$ indicates that there is no difference between $x_{i}$ and $x_{j}$. $r_{i j}=1$ indicates that $x_{i}$ is absolutely preferred to $x_{j}$. $r_{i j}>\frac{1}{2}$ indicates that $x_{i}$ is preferred to $x_{j}$.

In this case, the preference matrix $M(\tilde{R})$ is usually assumed to be an additive reciprocal. i.e. $r_{i j}+r_{j i}=1$, $\forall i, j \in\{1, \cdots, n\}$.

Definition 2.2.2. [14] $\tilde{R}$ is called weak reflexive, if $\tilde{R}\left(x_{i}, x_{i}\right)=\frac{1}{2} \quad\left(\forall x_{i} \in U\right)$.

\section{Fuzzy Rough Set Model for Interval and Set-Valued Information Systems}

Definition 3.1. Let $S=(U, A, V, f)$ be an interval and set-valued information system, $\forall a \in A$, a fuzzy relation $\tilde{R}$ can be defined as:

$$
=\frac{\tilde{R}_{a}\left(x_{i}, x_{j}\right)}{\left|\left\{x_{k} \mid \min \left(f_{a}^{+}\left(x_{k}\right)\right) \geq \min \left(f_{a}^{+}\left(x_{i}\right)\right) \wedge \max \left(f_{a}^{-}\left(x_{k}\right)\right) \geq \max \left(f_{a}^{-}\left(x_{i}\right)\right)\right\}\right|+\left|\left\{x_{k} \mid \min \left(f_{a}^{+}\left(x_{k}\right)\right) \geq \min \left(f_{a}^{+}\left(x_{j}\right)\right) \wedge \max \left(f_{a}^{-}\left(x_{k}\right)\right) \geq \max \left(f_{a}^{-}\left(x_{j}\right)\right)\right\}\right|}
$$

For a set of attributes $B \subseteq A$, a fuzzy relation $\tilde{R}_{B}$ is defined as: $\tilde{R}_{B}\left(x_{i}, x_{j}\right)=\inf _{a \in B} \tilde{R}_{a}\left(x_{i}, x_{j}\right)$.

Obviously, there are some important properties of the fuzzy relation defined above:

1) $\tilde{R}_{a}\left(x_{i}, x_{i}\right)=\frac{1}{2}$, we know that $\tilde{R}_{a}$ is weak reflexive;

2) $\tilde{R}_{a}\left(x_{i}, x_{j}\right)+\tilde{R}_{a}\left(x_{j}, x_{i}\right)=1$, we know that $\tilde{R}_{a}$ is additive reciprocal.

Hence, $\tilde{R}$ is a fuzzy preference relation.

Definition 3.2. Let $S=(U, A, V, f)$ be an interval and set-valued information system, $\tilde{R}$ is a fuzzy preference relation on $U, \forall x_{i} \in U, B \subseteq A$, a fuzzy preference class $P_{\tilde{R}_{B}}\left(x_{i}\right)$ of $x_{i} \in U$ induced by the relation $\tilde{R}$ can be defined as:

$$
P_{\tilde{R}_{B}}\left(x_{i}\right)=\frac{r_{i 1}}{x_{1}}+\frac{r_{i 2}}{x_{2}}+\cdots+\frac{r_{i n}}{x_{n}} \quad\left(P_{\tilde{R}_{B}}\left(x_{i}\right)\right. \text { can also be }
$$

regarded as the fuzzy information granule), where $r_{i j}=\tilde{R}_{B}\left(x_{i}, x_{j}\right)$. Here, "+” means the unions of elements. $P_{\tilde{R}_{B}}\left(x_{i}\right)$ is a fuzzy set, and the fuzzy cardinal number of $P_{\tilde{R}_{B}}\left(x_{i}\right)$ is defined as: $\left|P_{\tilde{R}_{B}}\left(x_{i}\right)\right|=\sum_{j=1}^{n} r_{i j}$.

For a finite set $X, \forall x_{j} \in X$, we have $r_{i j} \leq 1$, then the cardinality of $P_{\tilde{R}_{B}}\left(x_{i}\right)$ is also finite and $\left|P_{\tilde{R}_{B}}\left(x_{i}\right)\right| \leq|U|$.

Definition 3.3. Give a fuzzy preference relation $\tilde{R}$ on $U . \forall \alpha \in[0,1]$, the $\alpha$-cuts is a crisp relation, where

$$
\tilde{R}_{\alpha}\left(x_{i}, x_{j}\right)= \begin{cases}1 & \tilde{R}\left(x_{i}, x_{j}\right) \geq \alpha \\ 0 & \tilde{R}\left(x_{i}, x_{j}\right)<\alpha\end{cases}
$$

Theorem 3.1. Let $S=(U, A, V, f)$ be an interval and set-valued information system, $\tilde{R}$ is a fuzzy preference relation on $U, \forall x_{i}, x_{j} \in U, B, C \subseteq A$, if $B \subseteq C$, then $\tilde{R}_{C} \subseteq \tilde{R}_{B}$ i.e. $\forall x_{i}, x_{j} \in U$, if $B \subseteq C$, then $\tilde{R}_{C}\left(x_{i}, x_{j}\right) \subseteq \tilde{R}_{B}\left(x_{i}, x_{j}\right)$.

Proof. It easy to prove according to Definition 3.1.

\section{Fuzzy Positive Region Reduct in Interval and Set-Valued Decision Information Systems}

In this section, we investigate fuzzy positive region reduct with respect to the fuzzy preference relation in interval and set-valued decision information systems.

Definition 4.1. Given an information system $S=(U, A, f, d, g)$, where the universe $U=\left\{x_{1}, x_{2}, \cdots, x_{n}\right\}$ is a non-empty finite set of objects, $A=\left\{a_{1}, a_{2}, \cdots, a_{m}\right\}$ is a non-empty finite set of condition attributes, $d$ is a non-empty finite set of decision attributes, $f: U \rightarrow A$ is a function that assigns particular values from condition attribute domains to objects, $g: U \rightarrow d$ is a function that assigns particular values from decision attribute domains to objects, then the in- 
formation system is called an interval and set-valued decision information system.

Definition 4.2. Let $S=(U, A, f, d, g)$ be an interval and set-valued decision information system, $\tilde{R}$ is a fuzzy preference relation on $U, U / d=\left\{D_{1}, D_{2}, \cdots, D_{r}\right\}$, $D_{k} \in U / d, 1 \leq k \leq r$, then define two fuzzy operators as follows:

$$
\begin{gathered}
\underline{\tilde{R}}_{A}\left(D_{k}\right)\left(x_{i}\right)=\inf _{x_{j} \notin D_{k}}\left(1-\tilde{R}_{A}\left(x_{i}, x_{j}\right)\right) \\
\overline{\tilde{R}}_{A}\left(D_{k}\right)\left(x_{i}\right)=\sup _{x_{j} \in D_{k}}\left(\tilde{R}_{A}\left(x_{i}, x_{j}\right)\right)
\end{gathered}
$$

$\underline{\tilde{R}}_{A}\left(D_{k}\right)\left(x_{i}\right)$ and $\overline{\tilde{R}}_{A}\left(D_{k}\right)\left(x_{i}\right)$ are called fuzzy lower approximation operator and upper approximation operator of decision class $D_{k}$ with respect to $\tilde{R}$, respectively.

Theorem 4.1. Let $S=(U, A, f, d, g)$ be an interval and set-valued decision information system, $B, C \subseteq A$, then the following properties hold:

1) $\forall C \subseteq B$, we have $\underline{\tilde{R}}_{C}\left(D_{k}\right) \subseteq \underline{\tilde{R}}_{B}\left(D_{k}\right)$,

$$
\overline{\tilde{R}}_{B}\left(D_{k}\right) \subseteq \overline{\tilde{R}}_{C}\left(D_{k}\right) .
$$

2) $\underline{\tilde{R}}_{B \cap C}\left(D_{k}\right) \subseteq \underline{\tilde{R}}_{B}\left(D_{k}\right) \cap \underline{\tilde{R}}_{C}\left(D_{k}\right)$,

$$
\overline{\tilde{R}}_{B \cap C}\left(D_{k}\right) \supseteq \overline{\tilde{R}}_{B}\left(D_{k}\right) \cup \overline{\tilde{R}}_{C}\left(D_{k}\right) \text {. }
$$

Proof. Since $C \subseteq B$, according to Theorem 3.1, we know $\tilde{R}_{B}\left(x_{i}, x_{j}\right) \subseteq \tilde{R}_{C}\left(x_{i}, x_{j}\right)$. Therefore,

$$
\begin{gathered}
1-\tilde{R}_{C}\left(x_{i}, x_{j}\right) \leq 1-\tilde{R}_{B}\left(x_{i}, x_{j}\right) \\
\underline{\tilde{R}}_{C}\left(D_{k}\right)\left(x_{i}\right)=\inf _{x_{j} \notin D_{k}}\left(1-\tilde{R}_{C}\left(x_{i}, x_{j}\right)\right) \\
\leq \inf _{x_{j} \notin D_{k}}\left(1-\tilde{R}_{B}\left(x_{i}, x_{j}\right)\right)=\underline{\tilde{R}}_{B}\left(D_{k}\right)\left(x_{i}\right) \\
\overline{\tilde{R}}_{B}\left(D_{k}\right)\left(x_{i}\right)=\sup _{x_{j} \in D_{k}}\left(\tilde{R}_{B}\left(x_{i}, x_{j}\right)\right) \\
\leq \sup _{x_{j} \in D_{k}}\left(\tilde{R}_{C}\left(x_{i}, x_{j}\right)\right)=\overline{\tilde{R}}_{C}\left(D_{k}\right)\left(x_{i}\right)
\end{gathered}
$$

Therefore, the equation $\underline{\tilde{R}}_{C}\left(D_{k}\right) \subseteq \underline{\tilde{R}}_{B}\left(D_{k}\right)$ and $\overline{\tilde{R}}_{B}\left(D_{k}\right) \subseteq \tilde{\tilde{R}}_{C}\left(D_{k}\right)$ is proved.

2) Since $B \cap C \subseteq B, B \cap C \subseteq C$, then according to 1 ), we have $\underline{\tilde{R}}_{B \cap C}\left(D_{k}\right) \subseteq \underline{\tilde{R}}_{B}\left(D_{k}\right), \underline{\tilde{R}}_{B \cap C}\left(D_{k}\right) \subseteq \underline{\tilde{R}}_{C}\left(D_{k}\right)$,

Therefore, the equation $\underline{\tilde{R}}_{B \cap C}\left(D_{k}\right) \subseteq \underline{\tilde{R}}_{B}\left(D_{k}\right) \cap \underline{\tilde{R}}_{C}\left(D_{k}\right)$ is proved, and the equation

$\tilde{\tilde{R}}_{B \cap C}\left(D_{k}\right) \supseteq \tilde{\tilde{R}}_{B}\left(D_{k}\right) \cup \tilde{\tilde{R}}_{C}\left(D_{k}\right)$ can be proved in a similar way.

Definition 4.3. Let $S=(U, A, f, d, g)$ be an interval and set-valued decision information system and $B \subseteq A$, decision class $U / d=\left\{D_{1}, D_{2}, \cdots, D_{r}\right\}, D_{k} \in U / d$. Then the fuzzy positive region of $d$ with respect to $B$ is denoted by $\operatorname{POS}_{B}(d)$, the membership function is defined by

$$
\operatorname{POS}_{B}(d)\left(x_{i}\right)=\sup _{D_{k} \in U / d} \underline{\tilde{R}}_{B}\left(D_{k}\right)\left(x_{i}\right) \quad \forall x_{i} \in U
$$

With respect to $B \subseteq A$, according to Theorem 4.1, we have $\underline{\tilde{R}}_{B}\left(D_{k}\right) \subseteq \underline{\tilde{R}}_{A}\left(D_{k}\right)$, hence, $\operatorname{POS}_{B}(d) \subseteq \operatorname{POS}_{A}(d)$.

Definition 4.4. Let $S=(U, A, f, d, g)$ be an interval and set-valued decision information system and $a \in B \subseteq A$. If $\operatorname{POS}_{B-\{a\}}(d)=\operatorname{POS}_{B}(d)$, then the attribute $a$ in $B$ is dispensable. Otherwise, the attribute $a$ in $B$ is indispensable. If each attribute of $B$ is indispensable, then $B$ is called independent. All indispensable attributes of $A$ is called the core of interval and set-valued information system, which is denoted by Core $(S)$.

Definition 4.5. Let $S=(U, A, f, d, g)$ be an interval and set-valued decision information system and $B \subseteq A$, $B$ is a fuzzy positive region reduct of $S$ if

1) $\operatorname{POS}_{B}(d)=\operatorname{POS}_{A}(d)$,

2) $\forall a \in B, \operatorname{POS}_{B-\{a\}}(d) \neq \operatorname{POS}_{B}(d)$.

Fuzzy positive region reduct in interval and set-valued decision information systems is the minimal attribute subset that keeps positive region invariant, and it is easy to prove that for any fuzzy positive region reduct $B$ of $S$, we have $\operatorname{Core}(S) \subseteq B$.

Generally speak, information system $S$ may have many reducts, all the reducts of $S$ is denoted by $\operatorname{Red}(S)$. According to the definition of core, it is easy to get the following theorem:

Theorem 4.2. Let $S=(U, A, f, d, g)$ be an interval and set-valued decision information system, then we have Core $(S)=\bigcap \operatorname{Red}(S)$, i.e. Core $(S)$ is the intersection of all reducts of $S$.

Definition 4.6. Let $S=(U, A, f, d, g)$ be an interval and set-valued decision information system and $B \subseteq A$. Then the dependency degree of $d$ to $B$ is defined by

$$
\gamma_{B}(d)=\frac{\sum_{x_{i} \in U} \operatorname{POS}_{B}(d)\left(x_{i}\right)}{|U|} \quad \forall x_{i} \in U
$$

Obviously, $0 \leq \gamma_{B}(d) \leq 1$, and with respect to $B \subseteq A$, we have $\gamma_{B}(d) \leq \gamma_{A}(d)$.

Definition 4.7. Let $S=(U, A, f, d, g)$ be an interval and set-valued decision information system. If $\gamma_{A}(d)=1$, then $S$ is called a consistent decision system; otherwise, it is referred to as an inconsistent decision system.

Theorem 4.3. Let $S=(U, A, f, d, g)$ be an interval and set-valued decision information system and $B \subseteq A$, then $B$ is a fuzzy positive region reduct of $S$ if and only if

1) $\gamma_{B}(d)=\gamma_{A}(d)$,

2) $\forall a \in B, \gamma_{B}(d)>\gamma_{B-\{a\}}(d)$.

Proof. The necessity of obvious, we prove the sufficiency in the following:

If $\operatorname{POS}_{B}(d) \neq \operatorname{POS}_{A}(d)$, according to Theorem 4.1 and Definition 4.3, $\exists x_{i} \in U$, such that 
$\operatorname{POS}_{B}(d)\left(x_{i}\right)<\operatorname{POS}_{A}(d)\left(x_{i}\right)$, then $\gamma_{B}(d)<\gamma_{A}(d)$, which contradicts $\gamma_{B}(d)=\gamma_{A}(d)$. On the other hand, If $\exists a \in B$, such that $a$ is dispensable, then we have $\operatorname{POS}_{B-\{a\}}(d)=\operatorname{POS}_{B}(d), \quad \gamma_{B}(d)=\gamma_{B-\{a\}}(d)$, which contradicts $\gamma_{B}(d)>\gamma_{B-\{a\}}(d)$. Therefore $B$ is a fuzzy positive region reduct of $S$. Hence, we complete the proof of Theorem 4.3.

Definition 4.8. Let $S=(U, A, f, d, g)$ be an interval and set-valued decision information system and $B \subseteq A$, $\forall a \in B$, the significance measure of $a$ in $B$ is defined as:

$$
\operatorname{Sig}(a, B, d)=\gamma_{B}(d)-\gamma_{B-\{a\}}(d)
$$

Theorem 4.4. Let $S=(U, A, f, d, g)$ be an interval and set-valued decision information system, then the core attribute of $S$ satisfies

$$
\operatorname{Core}(S)=\{a \in A: \operatorname{Sig}(a, A, d)>0\}
$$

\section{Proof.}

$$
a \in \operatorname{Core}(S) \Leftrightarrow \gamma_{A}(d)>\gamma_{A-\{a\}}(d) \Leftrightarrow \operatorname{Sig}(a, A, d)>0
$$

Definition 4.9. Let $S=(U, A, f, d, g)$ be an interval andset-valued decision information system, $B \subseteq A$, $a \in A-B$, then the relative significance measure of attribute $a$ to $B$ is defined as

$$
\operatorname{Sig}_{B}(a, d)=\gamma_{B \cup\{a\}}(d)-\gamma_{B}(d)
$$

Give an interval and set-valued decision information system $S=(U, A, f, d, g)$, according to the significance measure and the relative significance measure, we can get a calculation method of fuzzy positive region reduct. The specific steps are written as follows:

Algorithm. Fuzzy positive reduct in interval and setvalued decision information systems.

Input: An interval and set-valued decision information system $S=(U, A, f, d, g)$.

Output: Fuzzy positive reduct of interval and setvalued decision information system $S=(U, A, f, d, g)$.

Step 1. Compute the dependency degree $\gamma_{A}(d)$,

Step 2. $\forall a \in A$, compute $\operatorname{Sig}(a, A, d)$, Core $(S)=\{a \in A: \operatorname{Sig}(a, A, d)>0\}$, If

$\gamma_{\text {Core }(S)}(d)=\gamma_{A}(d)$, then $\operatorname{Core}(S)$ is a fuzzy positive region reduct of $S$. Otherwise, we need to go to Step 3 .

Step 3. Let $B=\operatorname{Core}(S)$, with respect to condition attribute subset $A-B$, cycle the following steps:

1) $\forall a \in A-B$, compute relative significance measure $\operatorname{Sig}_{B}(a, d)$.

2) Choose $\forall a_{0} \in A-B$, such that

$\operatorname{Sig}_{B}\left(a_{0}, d\right)=\max _{a \in A-B} \operatorname{Sig}_{B}(a, d)$ and make $B=B \bigcup\left\{a_{0}\right\}$.

3) If $\gamma_{B}(d)=\gamma_{A}(d)$, then $B$ is fuzzy positive region reduct of $S$. Otherwise, return to 1$)$.

\section{Illustrative Example}

Example. Table 1 depicts an interval and set-valued decision information system containing information about risk investment of a company, where

$U=\left\{x_{1}, x_{2}, x_{3}, x_{4}, x_{5}\right\}$ denotes five companies,

$A=\left\{a_{1}, a_{2}, a_{3}, a_{4}, a_{5}\right\}=$ \{market risk, technology risk, operational risk, environmental risk, product risk, financial risk , and $d$ is the decision attribute.

By Definition 3.1, we can know.

\begin{tabular}{|c|c|c|c|c|c|c|c|}
\hline$U$ & $a_{1}$ & $a_{2}$ & $a_{3}$ & $a_{4}$ & $a_{5}$ & $a_{6}$ & $d$ \\
\hline$x_{1}$ & $V_{\{3\}}^{\{4\}}$ & $V_{\{4\}}^{\{5\rangle}$ & $V_{\{3\}}^{\{4\}}$ & $V_{\{3\}}^{\{4\}}$ & $V_{\{2\}}^{\{3\}}$ & $V_{\langle 4\}^{(5)}}^{(5 ;}$ & 1 \\
\hline$x_{2}$ & $V_{\{1\}}^{\{2\}}$ & $V_{\{1\}}^{\{2\}}$ & $V_{\{1\}}^{\{2,3\}}$ & $V_{\{1\}}^{\{2,3\}}$ & $V_{\{2\}}^{\{3\}}$ & $V_{\{1,1\}}^{\{2,3]}$ & 2 \\
\hline$x_{3}$ & $V_{\{3\}}^{\{4\}}$ & $V_{\{4\}}^{\{5\}}$ & $V_{\{3,4\}}^{\{5\}}$ & $V_{\{3\}}^{\{4\}}$ & $V_{\{3\}}^{\{4,5\}}$ & $V_{\{4\}}^{[s\}}$ & 1 \\
\hline$x_{4}$ & $V_{\{2\}}^{\{3\}}$ & $V_{\{4\}}^{\{5\}}$ & $V_{\{2\}}^{\{3\}}$ & $V_{\{2,3\}}^{\{3,4\}}$ & $V_{\{2\}}^{\{3\}}$ & $V_{\{3\}}^{\{4,5\}}$ & 2 \\
\hline$x_{5}$ & $V_{\{1\}}^{\{2\}}$ & $V_{\{1\}}^{\{2\}}$ & $V_{\{1\}}^{\{2,3\}}$ & $V_{\{1\}}^{\{2\}}$ & $V_{\{2\}}^{\{3\}}$ & $V_{\{1,2\}}^{\{3\}}$ & 2 \\
\hline
\end{tabular}

$$
\begin{aligned}
& M\left(\tilde{R}_{a_{1}}\right)=\left(\begin{array}{ccccc}
\frac{1}{2} & \frac{2}{7} & \frac{1}{2} & \frac{2}{5} & \frac{2}{7} \\
\frac{5}{7} & \frac{1}{2} & \frac{5}{7} & \frac{5}{8} & \frac{1}{2} \\
\frac{1}{2} & \frac{2}{7} & \frac{1}{2} & \frac{2}{5} & \frac{2}{7} \\
\frac{3}{5} & \frac{3}{8} & \frac{3}{5} & \frac{1}{2} & \frac{3}{8} \\
\frac{5}{7} & \frac{1}{2} & \frac{5}{7} & \frac{5}{8} & \frac{1}{2}
\end{array}\right) \\
& M\left(\tilde{R}_{a_{2}}\right)=\left(\begin{array}{ccccc}
\frac{1}{2} & \frac{3}{8} & \frac{1}{2} & \frac{1}{2} & \frac{3}{8} \\
\frac{5}{8} & \frac{1}{2} & \frac{5}{8} & \frac{5}{8} & \frac{1}{2} \\
\frac{1}{2} & \frac{3}{8} & \frac{1}{2} & \frac{1}{2} & \frac{3}{8} \\
\frac{1}{2} & \frac{3}{8} & \frac{1}{2} & \frac{1}{2} & \frac{3}{8} \\
\frac{5}{8} & \frac{1}{2} & \frac{5}{8} & \frac{5}{8} & \frac{1}{2}
\end{array}\right) \\
& M\left(\tilde{R}_{a_{3}}\right)=\left(\begin{array}{lllll}
\frac{1}{2} & \frac{2}{7} & \frac{2}{3} & \frac{2}{5} & \frac{2}{7} \\
\frac{5}{7} & \frac{1}{2} & \frac{5}{6} & \frac{5}{8} & \frac{1}{2} \\
\frac{1}{3} & \frac{1}{6} & \frac{1}{2} & \frac{1}{4} & \frac{1}{6} \\
\frac{3}{5} & \frac{3}{8} & \frac{3}{4} & \frac{1}{2} & \frac{3}{8} \\
\frac{5}{7} & \frac{1}{2} & \frac{5}{6} & \frac{5}{8} & \frac{1}{2}
\end{array}\right)
\end{aligned}
$$

Table 1. An interval and set-valued decision information system. 


$$
\begin{aligned}
& M\left(\tilde{R}_{a_{4}}\right)=\left(\begin{array}{ccccc}
\frac{1}{2} & \frac{2}{7} & \frac{1}{2} & \frac{2}{5} & \frac{2}{7} \\
\frac{5}{7} & \frac{1}{2} & \frac{5}{7} & \frac{5}{8} & \frac{1}{2} \\
\frac{1}{2} & \frac{2}{7} & \frac{1}{2} & \frac{2}{5} & \frac{2}{7} \\
\frac{3}{5} & \frac{3}{8} & \frac{3}{5} & \frac{1}{2} & \frac{3}{8} \\
\frac{5}{7} & \frac{1}{2} & \frac{5}{7} & \frac{5}{8} & \frac{1}{2}
\end{array}\right) \\
& M\left(\tilde{R}_{a_{5}}\right)=\left(\begin{array}{ccccc}
\frac{1}{2} & \frac{1}{2} & \frac{5}{6} & \frac{1}{2} & \frac{1}{2} \\
\frac{1}{2} & \frac{1}{2} & \frac{5}{6} & \frac{1}{2} & \frac{1}{2} \\
\frac{1}{6} & \frac{1}{6} & \frac{1}{2} & \frac{1}{6} & \frac{1}{6} \\
\frac{1}{2} & \frac{1}{2} & \frac{5}{6} & \frac{1}{2} & \frac{1}{2} \\
\frac{1}{2} & \frac{1}{2} & \frac{5}{6} & \frac{1}{2} & \frac{1}{2}
\end{array}\right) \\
& M\left(\tilde{R}_{a_{6}}\right)=\left(\begin{array}{ccccc}
\frac{1}{2} & \frac{2}{7} & \frac{1}{2} & \frac{2}{5} & \frac{1}{3} \\
\frac{5}{7} & \frac{1}{2} & \frac{5}{7} & \frac{5}{8} & \frac{5}{9} \\
\frac{1}{2} & \frac{2}{7} & \frac{1}{2} & \frac{2}{5} & \frac{1}{3} \\
\frac{3}{5} & \frac{3}{8} & \frac{3}{5} & \frac{1}{2} & \frac{3}{7} \\
\frac{2}{3} & \frac{4}{9} & \frac{2}{3} & \frac{4}{7} & \frac{1}{2}
\end{array}\right)
\end{aligned}
$$

According to Definition 3.1

$\tilde{R}_{A}\left(x_{i}, x_{j}\right)=\inf _{a \in A} \tilde{R}_{a}\left(x_{i}, x_{j}\right)$,

We have $M\left(\tilde{R}_{A}\right)=\left(\begin{array}{ccccc}\frac{1}{2} & \frac{2}{7} & \frac{1}{2} & \frac{2}{5} & \frac{2}{7} \\ \frac{1}{2} & \frac{1}{2} & \frac{5}{8} & \frac{1}{2} & \frac{1}{2} \\ \frac{1}{6} & \frac{1}{6} & \frac{1}{2} & \frac{1}{6} & \frac{1}{6} \\ \frac{1}{2} & \frac{3}{8} & \frac{1}{2} & \frac{1}{2} & \frac{3}{8} \\ \frac{1}{2} & \frac{4}{9} & \frac{5}{8} & \frac{1}{2} & \frac{1}{2}\end{array}\right)$

Then $\left|P_{\tilde{R}_{A}}\left(x_{1}\right)\right|=\frac{69}{35},\left|P_{\tilde{R}_{A}}\left(x_{2}\right)\right|=\frac{21}{8},\left|P_{\tilde{R}_{A}}\left(x_{3}\right)\right|=\frac{7}{6}$, $\left|P_{\tilde{R}_{A}}\left(x_{4}\right)\right|=\frac{9}{4},\left|P_{\tilde{R}_{A}}\left(x_{5}\right)\right|=\frac{185}{72}$.
1) Computer the dependency degree

$$
D_{1}=\left\{x_{1}, x_{3}\right\}, D_{2}=\left\{x_{2}, x_{4}, x_{5}\right\}
$$

According to Definition 4.2, we have

$$
\begin{array}{ll}
\underline{\tilde{R}}_{A}\left(D_{1}\right)\left(x_{1}\right)=\frac{3}{5} & \underline{\tilde{R}}_{A}\left(D_{2}\right)\left(x_{1}\right)=\frac{1}{2} \\
\underline{\tilde{R}}_{A}\left(D_{1}\right)\left(x_{2}\right)=\frac{1}{2} & \underline{\tilde{R}}_{A}\left(D_{2}\right)\left(x_{2}\right)=\frac{3}{8} \\
\underline{\tilde{R}}_{A}\left(D_{1}\right)\left(x_{3}\right)=\frac{5}{6} & \underline{\tilde{R}}_{A}\left(D_{2}\right)\left(x_{3}\right)=\frac{1}{2} \\
\underline{\tilde{R}}_{A}\left(D_{1}\right)\left(x_{4}\right)=\frac{1}{2} & \underline{\tilde{R}}_{A}\left(D_{2}\right)\left(x_{4}\right)=\frac{1}{2} \\
\underline{\tilde{R}}_{A}\left(D_{1}\right)\left(x_{5}\right)=\frac{1}{2} & \underline{\tilde{R}}_{A}\left(D_{2}\right)\left(x_{5}\right)=\frac{3}{8}
\end{array}
$$

According to Definition 4.6, we have

$$
\begin{gathered}
\gamma_{A}(d)=\frac{1}{5}\left(\frac{3}{5}+\frac{1}{2}+\frac{5}{6}+\frac{1}{2}+\frac{1}{2}\right)=\frac{44}{75} \\
\gamma_{A-\left\{a_{1}\right\}}(d)=\frac{1}{5}\left(\frac{3}{5}+\frac{1}{2}+\frac{5}{6}+\frac{1}{2}+\frac{1}{2}\right)=\frac{44}{75} \\
\gamma_{A-\left\{a_{2}\right\}}(d)=\frac{1}{5}\left(\frac{3}{5}+\frac{1}{2}+\frac{5}{6}+\frac{1}{2}+\frac{1}{2}\right)=\frac{44}{75} \\
\gamma_{A-\left\{a_{3}\right\}}(d)=\frac{1}{5}\left(\frac{3}{5}+\frac{1}{2}+\frac{5}{6}+\frac{1}{2}+\frac{1}{2}\right)=\frac{44}{75} \\
\gamma_{A-\left\{a_{4}\right\}}(d)=\frac{1}{5}\left(\frac{3}{5}+\frac{1}{2}+\frac{5}{6}+\frac{1}{2}+\frac{1}{2}\right)=\frac{44}{75} \\
\gamma_{A-\left\{a_{5}\right\}}(d)=\frac{1}{5}\left(\frac{3}{5}+\frac{3}{8}+\frac{3}{4}+\frac{1}{2}+\frac{3}{7}\right)=\frac{743}{1400} \\
\gamma_{A-\left\{a_{6}\right\}}(d)=\frac{1}{5}\left(\frac{3}{5}+\frac{1}{2}+\frac{5}{6}+\frac{1}{2}+\frac{1}{2}\right)=\frac{44}{75}
\end{gathered}
$$

(According to Definition 4.7, since $\gamma_{A}(d)=\frac{1}{5}\left(\frac{3}{5}+\frac{1}{2}+\frac{5}{6}+\frac{1}{2}+\frac{1}{2}\right)=\frac{44}{75} \neq 1$, so this decision information system is an inconsistent decision system.)

2) Compute $\operatorname{Core}(S)$ (the significance measure of each attribute)

According to Definition 4.8, we have

$$
\begin{gathered}
\operatorname{Sig}\left(a_{1}, A, d\right)=0 \quad \operatorname{Sig}\left(a_{2}, A, d\right)=0 \\
\operatorname{Sig}\left(a_{3}, A, d\right)=0 \quad \operatorname{Sig}\left(a_{4}, A, d\right)=0 \\
\operatorname{Sig}\left(a_{5}, A, d\right)=\frac{47}{84}>0 \quad \operatorname{Sig}\left(a_{6}, A, d\right)=0
\end{gathered}
$$

Therefore, Core $(S)=\left\{a_{5}\right\}, \gamma_{\text {Core }(S)}(d)=\frac{17}{30} \neq \gamma_{A}(d)$.

3) Compute the relative significance measure 
Let $B=$ Core $(S)=\left\{a_{5}\right\}$, according to Definition 4.9, we have

$$
\begin{aligned}
& \operatorname{Sig}_{B}\left(a_{1}, d\right)=\gamma_{B \cup\left\{a_{1}\right\}}(d)-\gamma_{B}(d)=\frac{44}{75}-\frac{17}{30}=\frac{1}{50} \\
& \operatorname{Sig}_{B}\left(a_{2}, d\right)=\gamma_{B \cup\left\{a_{2}\right\}}(d)-\gamma_{B}(d)=\frac{17}{30}-\frac{17}{30}=0 \\
& \operatorname{Sig}_{B}\left(a_{3}, d\right)=\gamma_{B \cup\left\{a_{3}\right\}}(d)-\gamma_{B}(d)=\frac{44}{75}-\frac{17}{30}=\frac{1}{50} \\
& \operatorname{Sig}_{B}\left(a_{4}, d\right)=\gamma_{B \cup\left\{a_{4}\right\}}(d)-\gamma_{B}(d)=\frac{44}{75}-\frac{17}{30}=\frac{1}{50} \\
& \operatorname{Sig}_{B}\left(a_{5}, d\right)=\gamma_{B \cup\left\{a_{5}\right\}}(d)-\gamma_{B}(d)=\frac{44}{75}-\frac{17}{30}=\frac{1}{50} \\
& \operatorname{Sig}_{B}\left(a_{6}, d\right)=\gamma_{B \cup\left\{a_{6}\right\}}(d)-\gamma_{B}(d)=\frac{44}{75}-\frac{17}{30}=\frac{1}{50} \\
& \gamma_{\left\{a_{1}, a_{5}\right\}}(d)=\frac{1}{5}\left(\frac{3}{5}+\frac{1}{2}+\frac{5}{6}+\frac{1}{2}+\frac{1}{2}\right)=\frac{44}{75}=\gamma_{A}(d) \\
& \gamma_{\left\{a_{3}, a_{5}\right\}}(d)=\frac{1}{5}\left(\frac{3}{5}+\frac{1}{2}+\frac{5}{6}+\frac{1}{2}+\frac{1}{2}\right)=\frac{44}{75}=\gamma_{A}(d) \\
& \gamma_{\left\{a_{4}, a_{5}\right\}}(d)=\frac{1}{5}\left(\frac{3}{5}+\frac{1}{2}+\frac{5}{6}+\frac{1}{2}+\frac{1}{2}\right)=\frac{44}{75}=\gamma_{A}(d) \\
& \gamma_{\left\{a_{5}, a_{6}\right\}}(d)=\frac{1}{5}\left(\frac{3}{5}+\frac{1}{2}+\frac{5}{6}+\frac{1}{2}+\frac{1}{2}\right)=\frac{44}{75}=\gamma_{A}(d)
\end{aligned}
$$

Hence, $\left\{a_{1}, a_{5}\right\},\left\{a_{3}, a_{5}\right\},\left\{a_{4}, a_{5}\right\}$ and $\left\{a_{5}, a_{6}\right\}$ are the fuzzy positive region reducts in interval and set-valued decision information system. We can conclude that product risk is the core influencing factor and market risk, operational risk, environmental risk and financial risk are the important influencing factors.

\section{Conclusions}

It is well known that attribute reduction is a basic issue in rough set theory. Recently, the attribute reduction based on fuzzy rough set in decision information systems has attracted the attention of many scholars.

In this paper, we introduce a fuzzy rough set model for interval and set-valued information systems by defining a fuzzy preference relation. The concepts of the significance measure of condition attributes and the relative significance measure of condition attributes are given in interval and set-valued decision information systems by the introduction of fuzzy positive region and the dependency degree. And on this basis, a heuristic algorithm for calculating fuzzy positive region reduction in interval and set-valued decision information systems is given.

The results will help us to gain much more insights into the meaning of fuzzy rough set theory. Furthermore, it has provided a new perspective to study the attribute reduction problem in decision systems.

\section{Acknowledgements}

This work is supported by the Natural Science Foundation of Shanxi Province in China (No. 2008011012).

\section{REFERENCES}

[1] Z. Pawlak, "Rough Sets: Theoretical Aspects of Reasoning about Data,” Kluwer Academic Publishers, Dordrecht, 1991.

[2] J. H. Dai and H. W. Tian, "Fuzzy Rough Set Model Forset-Valued Data,” Fuzzy Sets and Systems, Vol. 229, 2013, pp. 54-68. http://dx.doi.org/10.1016/j.fss.2013.03.005

[3] Y. H. Qian, J. Y. Liang, W. Z. Wu and C. Y. Dang, "Information Granularity in Fuzzy Binary Grc Model,” IEEE Transactions on Fuzzy Systems, Vol. 19, No. 2, 2011, pp. 253-264. http://dx.doi.org/10.1109/TFUZZ.2010.2095461

[4] Z. Q. Meng and Z. Z. Shi, "Extended Rough Set-Based Attribute Reduction in Inconsistent Incomplete Decision Systems,” Information Sciences, Vol. 204, 2012, pp. 4469. http://dx.doi.org/10.1016/j.ins.2012.04.004

[5] T. Q. Deng, C. D. Yang and X. F. Wang, "A Reduct Derived from Feature Selection," Pattern Recognition Letters, Vol. 33, No. 12, 2012, pp. 1638-1646. http://dx.doi.org/10.1016/j.patrec.2012.03.028

[6] Y. J. Lin, J. J. Li and S. X. Wu, "Rough Set Theory in Interval and Set-Valued Information Systems," Control and Decision, Vol. 26, No. 11, 2011, pp. 1611-1615.

[7] R. C. Berredo, P. Y. Ekel and R. M. Palhares, "Fuzzy Preference Relations in Models of Decision Making," Nonlinear Analysis, Vol. 63, No. 5-7, 2005, pp. e735-e741. http://dx.doi.org/10.1016/j.na.2005.02.093

[8] E. Herrera-Viedma, F. Herrera, F. Chiclana and M. Luque, "Some Issues on Consistency of Fuzzy Preference Relations,” European Journal of Operational Researcher, Vol. 154, No. 1, 2004, pp. 98-109. http://dx.doi.org/10.1016/S0377-2217(02)00725-7

[9] Q. H. Hu, D. R. Yu and M. Z. Guo, "Fuzzy Preference Based Rough Sets,” Information Sciences, Vol. 180, No. 10, 2010, pp. 2003-2022. http://dx.doi.org/10.1016/j.ins.2010.01.015

[10] Y. M. Wang and Z, P. Fan, "Fuzzy Preference Relations Aggregation and Weight Determination,” Computers \& Industrial Engineering, Vol. 53, No. 1, 2007, pp. 163172. http://dx.doi.org/10.1016/j.cie.2007.05.001

[11] L. L. Lee, “Group Decision Making with Incomplete Fuzzy Preference Relations Based on the Additive Consistency and the Other Consistency Original,” Experts Systems with Applications, Vol. 39, No. 14, 2012, pp. 11666-11676. http://dx.doi.org/10.1016/j.eswa.2012.04.043

[12] B. Zhu, "Studies on Consistency Measure of Hesitant Fuzzy Preference Relations,” Procedia Computer Science, Vol. 17, 2013, pp. 457-464. http://dx.doi.org/10.1016/j.procs.2013.05.059

[13] Y. H. Qian and J. Y. Liang, "Positive Approximation: An Accelerator for Attribute Reduction in Rough Set Theory,” Artificial Intelligence, Vol. 174, No. 9-10, 2010, pp. 597-618. http://dx.doi.org/10.1016/j.artint.2010.04.018 
[14] W. X. Zhang and G. F. Qiu, "Uncertain Decision Making Based on Rough Sets,” Qinghua Publishing Company, Beijing, 2005, pp. 133-144.

[15] D. R. Yu, Q. H. Hu and C. X. Wu, “Uncertainty Measures for Fuzzy Relations and Their Applications,” Applied Soft Computing, Vol. 7, No. 3, 2007, pp. 1135-1143. http://dx.doi.org/10.1016/j.asoc.2006.10.004

[16] Y. Q. Yao and J. S. Mi, “Attribute Reduction Based on Generalized Fuzzy Evidence Theory in Fuzzy Decision Systems,” Fuzzy Sets and Systems, Vol. 170, No. 1, 2011, pp. 64-75. http://dx.doi.org/10.1016/j.fss.2011.01.008

[17] R. Jensen and Q. Shen, "Fuzzy-Rough Attributes Reduction with Application to Web Categorization,” Fuzzy Sets and Systems, Vol. 141, No. 3, 2004, pp. 469-485. http://dx.doi.org/10.1016/S0165-0114(03)00021-6

[18] Q. H. Hu, D. R. Yu and Z. X. Xie, "Information-Preserving Hybrid Data Reduction Based on Fuzzy Rough Techniques,” Pattern Recognition Letters, Vol. 27, No. 5, 2006, pp. 414-423.

http://dx.doi.org/10.1016/j.patrec.2005.09.004
[19] B. Q. Hu and Y. X. Xian, "Level Characteristics of Rough Fuzzy Sets and Fuzzy Rough Sets,” Fuzzy Systems and Mathematics, Vol. 20, No. 1, 2006, pp.108-114.

[20] D. Dubois and H. Prade, "Rough Fuzzy Sets and Fuzzy Rough Sets,” International Journal of General Systems, Vol. 17, No. 2-3, 1990, pp. 191-209. http://dx.doi.org/10.1080/03081079008935107

[21] D. Dubois and H. Prade, "Putting Rough Sets and Fuzzy Sets Together,” Intelligent Decision Support, Vol. 11, 1992, pp. 203-232.

http://dx.doi.org/10.1007/978-94-015-7975-9_14

[22] R. B. Bhatt and M. Gopal, "On Fuzzy-Rough Sets Approach to Feature Selection,” Pattern Recognition Letters, Vol. 26, No. 7, 2005, pp. 965-975. http://dx.doi.org/10.1016/j.patrec.2004.09.044

[23] S. Y. Zhao, "On Fuzzy Approximation Operators in Attribute Reduction with Fuzzy Rough Sets,” Information Sciences, Vol. 178, No. 16, 2008, pp. 3163-3176. http://dx.doi.org/10.1016/j.ins.2008.03.022 